\title{
Algebrization of Nonautonomous Differential Equations
}

\author{
María Aracelia Alcorta-García, ${ }^{1}$ Martín Eduardo Frías-Armenta, ${ }^{2}$ \\ María Esther Grimaldo-Reyna, ${ }^{1}$ and Elifalet López-González ${ }^{3}$ \\ ${ }^{1}$ Universidad Autónoma de Nuevo León, Avenida Universidad, S/N, Ciudad Universitaria, 66451 San Nicolás de los Garza, NL, Mexico \\ ${ }^{2}$ Departamento de Matemáticas, Universidad de Sonora, Boulevard Rosales y Luis Encinas, S/N, Col. Centro, \\ 83000 Hermosillo, SON, Mexico \\ ${ }^{3}$ Universidad Autónoma de Ciudad Juárez, Unidad Multidisciplinaria de la UACJ en Cuauhtémoc, \\ Carretera Cuauhtémoc-Anáhuac, Col. Ejido Anáhuac km 3.5, S/N 31600, Municipio de Cuauhtémoc, CHIH, Mexico
}

Correspondence should be addressed to María Esther Grimaldo-Reyna; maria.grimaldory@uanl.edu.mx

Received 4 June 2015; Revised 22 September 2015; Accepted 11 October 2015

Academic Editor: Peter G. L. Leach

Copyright (c) 2015 María Aracelia Alcorta-García et al. This is an open access article distributed under the Creative Commons Attribution License, which permits unrestricted use, distribution, and reproduction in any medium, provided the original work is properly cited.

Given a planar system of nonautonomous ordinary differential equations, $d w / d t=F(t, w)$, conditions are given for the existence of an associative commutative unital algebra $A$ with unit $e$ and a function $H: \Omega \subset \mathbb{R}^{2} \times \mathbb{R}^{2} \rightarrow \mathbb{R}^{2}$ on an open set $\Omega$ such that $F(t, w)=H(t e, w)$ and the maps $H_{1}(\tau)=H(\tau, \xi)$ and $H_{2}(\xi)=H(\tau, \xi)$ are Lorch differentiable with respect to $\mathbb{A}$ for all $(\tau, \xi) \in \Omega$, where $\tau$ and $\xi$ represent variables in $\mathbb{A}$. Under these conditions the solutions $\xi(\tau)$ of the differential equation $d \xi / d \tau=H(\tau, \xi)$ over A define solutions $(x(t), y(t))=\xi(t e)$ of the planar system.

\section{Introduction}

The theory of analytic functions on algebras is based on Lorch analyticity; see [1-5]. Results of classical function theory have been extended to finite dimensional associative commutative unital algebras:

(i) The Cauchy integral theorem is satisfied for analytical functions in algebras, and the Cauchy integral formula has an analogous version in algebras.

(ii) The classical theorems on Taylor power series are easily established, and Laurent expansion may be defined in several disjoint regions in each one of which it may define a different analytic function.

(iii) Analyticity of functions of variables in algebras is characterized by the generalized Cauchy-Riemann equations, which is a set of first-order linear partial differential equations.
This theory allows us to consider differential equations over algebras, which can be used to solve family planar systems having the form

$$
\begin{aligned}
& \frac{d x}{d t}=f(t, x, y), \\
& \frac{d y}{d t}=g(t, x, y) .
\end{aligned}
$$

For this work and hereinafter any algebra will be assumed to be associative, commutative, and unital with unit $e$, and A will denote the linear space $\mathbb{R}^{2}$ endowed with an algebra structure.

In this paper a planar vector field $F$ is said to be $A$ algebrizable or $\mathbb{A}$-differentiable if there exists an algebra $\mathbb{A}$ for the which $F$ is Lorch differentiable (see Section 2 for definitions). In the same way, we say that a planar autonomous system of ordinary differential equations $d w / d t=F(w)$ is algebrizable if $F$ is A-algebrizable. 
Definition 1. Let $\mathbb{A}$ be an algebra. We say that a function $F: U \subset \mathbb{R}^{3} \rightarrow \mathbb{R}^{2}$ defined in an open set $U$ has an $\mathscr{H}(\mathbb{A})$ differentiable lifting $H: \Omega \subset \mathbb{R}^{2} \times \mathbb{R}^{2} \rightarrow \mathbb{R}^{2}$ if $H$ is a function defined in an open set $\Omega$ such that (i) the maps $H_{1}(\tau)=H(\tau, b)$ and $H_{2}(\xi)=H(a, \xi)$ are $\mathbb{A}$-differentiable functions with respect to $\mathbb{A}$ for all $(a, b) \in \Omega$, where $\tau$ and $\xi$ represent variables in $\mathbb{A}$, (ii) $(t e, x, y) \in \Omega$ for all $(t, x, y) \in U$, and (iii) $F(t, x, y)=H(t e, x, y)$ for all $(t, x, y) \in U$.

A nonautonomous differential equation over an algebra $\mathbb{A}$ is denoted by

$$
\frac{d \xi}{d \tau}=H(\tau, \xi)
$$

where $H: \Omega \subset \mathbb{A} \times \mathbb{A} \rightarrow \mathbb{A}$ is a function defined in an open set $\Omega$. For every point $\left(\tau_{0}, \xi_{0}\right) \in \Omega$, a solution to the equation through $\left(\tau_{0}, \xi_{0}\right)$ consists of an $\mathbb{A}$-differentiable function $\xi: N\left(\tau_{0}\right) \subset \mathbb{A} \rightarrow \mathbb{A}$ defined in a neighborhood $N\left(\tau_{0}\right)$ of $\tau_{0}$, with $\xi\left(\tau_{0}\right)=\xi_{0}$ and $\mathbb{A}$-derivative $d \xi / d \tau$ with respect to $\mathbb{A}$ satisfies $d \xi(\tau) / d \tau=H(\tau, \xi(\tau))$ for all $\tau \in N\left(\tau_{0}\right)$. If $F=(f, g)$ has an $\mathscr{H}(\mathbb{A})$-differentiable lifting $H$, we say that planar system (1) is algebrizable and that (2) is an algebrization of (1). A theorem of existence and uniqueness of solutions for differential equations over algebras is proved in [6], and a technique for visualization of solutions is given in [7].

The classical differential equation $d w / d t=w^{2}$ has solutions $w(t)=-(t+c)^{-1}$. Some differential systems of autonomous differential equations can be written in this form by using variables in algebras. For example, the algebrization of the planar differential system

$$
\begin{aligned}
& \frac{d x}{d t}=3 x^{2}+2 x y-y^{2} \\
& \frac{d y}{d t}=3 y^{2}+2 x y-x^{2}
\end{aligned}
$$

is the differential equation $d \xi / d \tau=\xi^{2}$ over the algebra $\mathbb{A}$ defined by the linear space $\mathbb{R}^{2}$ endowed with the product

$$
\begin{aligned}
& \left(x_{1}, y_{1}\right)\left(x_{2}, y_{2}\right):=\left(3 x_{1} x_{2}\right. \\
& \quad+\left(x_{1} y_{2}+y_{1} x_{2}-y_{1} y_{2}\right), 3 y_{1} y_{2} \\
& \left.\quad+\left(x_{1} y_{2}+y_{1} x_{2}-x_{1} x_{2}\right)\right) .
\end{aligned}
$$

The solutions $\xi$ are given by $\xi(\tau)=-(\tau+c)^{-1}$; hence the solutions of the planar system are given by $(x(t), y(t))=\xi(t e)$, where $e$ denotes the unit of $\mathbb{A}$. Using the first fundamental representation of $\mathbb{A}$ (see Section 2) the following expression for the solution $(x(t), y(t))$ of the system is obtained:

$$
\begin{aligned}
& (x(t), y(t)) \\
& \quad=\left(\frac{-t-y_{0}}{4\left(2 t+x_{0}+y_{0}\right)^{2}}, \frac{-t-x_{0}}{4\left(2 t+x_{0}+y_{0}\right)^{2}}\right), \\
& (x(0), y(0))=\frac{-\left(y_{0}, x_{0}\right)}{4\left(x_{0}+y_{0}\right)^{2}} .
\end{aligned}
$$

Consider now the planar nonautonomous differential system

$$
\begin{aligned}
& \frac{d x}{d t}=-\frac{1}{t^{3}}-\frac{2 x}{t}+t x^{2}-t y^{2} \\
& \frac{d y}{d t}=-\frac{2 y}{t}+2 t x y+t y^{2}
\end{aligned}
$$

whose algebrization is the Riccati differential equation over A:

$$
\frac{d \xi}{d \tau}=-\frac{1}{\tau^{3}}-\frac{2 \xi}{\tau}+\tau \xi^{2}
$$

where $\mathbb{A}$ is the algebra defined by $\mathbb{R}^{2}$ and the product $(u, v)(x, y)=(u x-v y, x y+v x+v y)$. By the classical Lie methods for solving differential equations (see [8-10]), the solutions of the Riccati equation have the form

$$
\xi(\tau)=\frac{C+\tau^{2}}{\tau^{2}\left(C-\tau^{2}\right)}, \quad C=(a, b) .
$$

The functions $(x(t), y(t))=\xi(t e)$, where $e$ is the unit of $\mathbb{A}$, are solutions of the planar system, which can be obtained via the first fundamental representation of $\mathbb{A}$ :

$$
\begin{aligned}
& (x(t), y(t))=\left(\frac{b^{2}+\left(a+b-t^{2}\right)\left(a+t^{2}\right)}{\left(a^{2}+a b+b^{2}\right) t^{2}-(2 a+b) t^{4}+t^{6}},\right. \\
& \left.\frac{-2 b}{\left(a^{2}+a b+b^{2}\right)-(2 a+b) t^{2}+t^{4}}\right) .
\end{aligned}
$$

We consider differential systems having the form (1), where $f, g: U \subset \mathbb{R}^{3} \rightarrow \mathbb{R}$ are $C^{1}$ functions defined in an open set $U$. The aim of this work is to give a family of functions $F=(f, g)$ having $\mathscr{H}(\mathbb{A})$-differentiable liftings $H$ over some algebra $\mathbb{A}$. When they exist, the solutions $\xi(\tau)$ of the differential equation $d \xi / d \tau=H(\tau, \xi)$ over $\mathbb{A}$ define solutions $(x(t), y(t))=\xi(t e)$ of system (1) which can be obtained via the first fundamental representation of $\mathbb{A}$.

The paper is organized as follows. In Section 2 definitions of algebra, algebrizability of planar vector fields, and differentiability on modules over algebras, a characterization of the algebrizability of planar vector fields and the form of all the quadratic vector fields which are algebrizable, are given. In Section 3 the definition of algebrizable liftings of functions $p: I \subset \mathbb{R} \rightarrow \mathbb{R}^{2}$ is presented. It is shown that the class of all of these functions defines an infinite dimensional algebra and the form of a family of these functions $p$ is given. In Section 4 it is proved that the solutions of planar systems (1) can be obtained from the solutions of their algebrization; a theorem containing conditions under which a planar system like (1) which is polynomial is algebrizable is given, and it is shown that the class of all the planar systems (1) having an algebrization (2) defines an infinite dimensional algebra. In Section 5 the case of quadratic systems is considered and their algebrizations are given, which are Riccati equations over algebras. The results presented in Sections 3, 4, and 5 are the main contributions of this paper. 


\section{Algebras and Lorch Analyticity}

\subsection{Algebras}

Definition 2 (see [11]). An algebra $\mathbb{A}$ is a $\mathbb{R}$-linear space $\mathbb{E}$ endowed with a bilinear product $\mathbb{A} \times \mathbb{A} \rightarrow \mathbb{A}$, denoted by $(x, y) \mapsto x y$, which is associative $x(y z)=(x y) z$ and commutative $x y=y x$ for all $x, y, z \in \mathbb{A}$, and has a unit $e \in \mathbb{A}$ satisfying ex $=x e=x$ for all $x \in \mathbb{A}$.

An element $a \in \mathbb{A}$ is called regular if there exists $a^{-1} \in \mathbb{A}$ called inverse of $a$ such that $a^{-1} \cdot a=a \cdot a^{-1}=e$. If $a \in \mathbb{A}$ is not regular, then $a$ is called singular. If $a, b \in \mathbb{A}$ and $b$ is regular, the quotient $a / b$ means $a \cdot b^{-1}$.

In all the algebras considered in this paper it will be the case that $\mathbb{E}=\mathbb{R}^{2}$, unless otherwise stated.

Consider an algebra $\mathbb{A}$. If $\beta=\left\{e_{1}, e_{2}\right\}$ is the standard basis of $\mathbb{R}^{2}$, the product between the elements of $\beta$ is given by $e_{i} e_{j}=\sum_{k=1}^{2} c_{i j k} e_{k}$, where the coefficients $c_{i j k} \in \mathbb{R}, i, j, k \in$ $\{1,2\}$, are called structure constants of $\mathbb{A}$. The first fundamental representation of $\mathbb{A}$ is the injective linear homomorphism $R$ : $\mathbb{A} \rightarrow M(2, \mathbb{R})$ defined by $R: e_{i} \mapsto R_{i}$, where $R_{i}$ is the matrix whose $j, k$ entry is $c_{i k j}$, for $i=1,2$.

2.2. Differentiability on Algebras. In this subsection the definition of Lorch differentiability is recalled, which in this paper is called $\mathbb{A}$-algebrizability or $\mathbb{A}$-differentiability to denote the dependence of the Lorch differential over an algebra $\mathbb{A}$.

Let $|\cdot|$ be the norm on $\mathbb{A}$ defined by $|a|=\max \{\|X R(a)\|$ : $\left.X \in \mathbb{R}^{2},\|X\|=1\right\}$ (here the vector $X$ is represented as a $1 \times 2$ matrix in order for the product $X R(a)$ to make sense), where $R: A \rightarrow M(2, R)$ is the first fundamental representation of $\mathbb{A}$ and $\|\cdot\|$ the Euclidean norm in $\mathbb{R}^{2}$. For this norm we have $|a b| \leq|a||b|$ for all $a, b \in M(2, \mathbb{R})$. Thus, we consider in this work that every algebra $\mathbb{A}$ is a Banach algebra under the norm $|\cdot|$.

Definition 3. Let $\mathbb{A}$ be an algebra and $F: V \subset \mathbb{A} \rightarrow \mathbb{A}$ a function on an open set $V$. We say that $F$ is $\mathbb{A}$-algebrizable or A-differentiable on $V$ if there exists a function $F^{\prime}: V \subset \mathbb{A} \rightarrow$ A, called the $\mathbb{A}$-derivative of $F$ on $V$, satisfying

$$
\lim _{h \rightarrow 0} \frac{\left|F(a+h)-F(a)-F^{\prime}(a) h\right|}{|h|}=0,
$$

for all $a \in V$, where $F^{\prime}(a) h$ denotes the product in $\mathbb{A}$ of $F^{\prime}(a)$ with $h$.

A vector field $F: V \subset \mathbb{A} \rightarrow \mathbb{A}$ is $\mathbb{A}$-algebrizable on $V$ if and only if the Jacobian matrix of $F$ is contained in the first fundamental representation of $\mathbb{A}$; that is, $J F(a) \in R(\mathbb{A})$ for all $a \in V$; see [5]. It can be shown that the notion of $\mathbb{A}$ algebrizability coincides with the holomorphicity when $\mathbb{A}$ is the complex field.

A method for determining whether a given planar vector field $F$ is algebrizable is the following. $F$ is algebrizable if and only if for some of the following three types of pairs of matrices

(I) $B_{1}=\left(\begin{array}{cc}1 & 0 \\ a & -1\end{array}\right), B_{2}=\left(\begin{array}{ll}0 & 1 \\ b & 0\end{array}\right)$,
(II) $B_{1}=\left(\begin{array}{cc}1 & a \\ 0 & -1\end{array}\right), B_{2}=\left(\begin{array}{ll}0 & 0 \\ 1 & 0\end{array}\right)$

(III) $B_{1}=\left(\begin{array}{ll}0 & 1 \\ 0 & 0\end{array}\right), B_{2}=\left(\begin{array}{ll}0 & 0 \\ 1 & 0\end{array}\right)$,

the condition $\left\langle B_{i}, \partial F(x, y) / \partial(x, y)\right\rangle=0$ is satisfied for $i=1,2$ and for all $(x, y)$ in the domain of definition of $F$, where $\langle\cdot, \cdot\rangle$ denotes the usual inner product in $M(2, \mathbb{R})$. The algebra for each type of pair of matrices is defined by the following corresponding product table of the standard basis vectors $e_{1}, e_{2}$ of $\mathbb{R}^{2}$ :

(I)

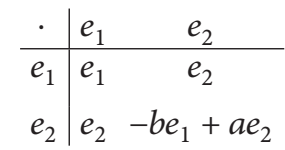

(II)

\begin{tabular}{c|cc}
$\cdot$ & $e_{1}$ & $e_{2}$ \\
\hline$e_{1}$ & $-a e_{1}$ & $e_{1}$ \\
$e_{2}$ & $e_{1}$ & $e_{2}$
\end{tabular}

(III)

\begin{tabular}{c|cc}
$\cdot$ & $e_{1}$ & $e_{2}$ \\
\hline$e_{1}$ & $e_{1}$ & 0 \\
$e_{2}$ & 0 & $e_{2}$
\end{tabular}

Consider a planar autonomous system of quadratic ordinary differential equations in the variables $x$ and $y$. If this system is algebrizable for an algebra with Type (I) product, then it can be represented by equations of the form

$$
\begin{aligned}
\frac{d x}{d t}= & a_{0}+\left(b_{2}-a b_{1}\right) x-b b_{1} y+\left(\frac{1}{2} b_{4}-a b_{3}\right) x^{2} \\
& -2 b b_{3} x y-\frac{1}{2} b b_{4} y^{2} \\
\frac{d y}{d t}= & b_{0}+b_{1} x+b_{2} y+b_{3} x^{2}+b_{4} x y \\
& +\left(\frac{1}{2} a b_{4}-b b_{3}\right) y^{2}
\end{aligned}
$$

for some real constants $a, b, a_{0}, b_{0}, b_{1}, \ldots, b_{4}$. In the case of algebrizability for an algebra with Type (II) product, the system is

$$
\begin{aligned}
& \frac{d x}{d t}=a_{0}+a_{1} x+a_{2} y-\frac{1}{2} a a_{3} x^{2}+a_{3} x y+a_{4} y^{2}, \\
& \frac{d y}{d t}=b_{0}+\left(a_{1}+a a_{2}\right) y+\left(\frac{1}{2} a_{3}+a a_{4}\right) y^{2},
\end{aligned}
$$

for some real constants $a, b_{0}, a_{0}, a_{1}, a_{2}, a_{3}$, and $a_{4}$. For Type (III) product the system can be represented by equations of the form

$$
\begin{aligned}
& \frac{d x}{d t}=a_{0}+a_{1} x+a_{2} x^{2}, \\
& \frac{d y}{d t}=b_{0}+b_{1} y+b_{2} y^{2}
\end{aligned}
$$

for some real constants $a_{i}, b_{i}, i=0,1,2$. Moreover, conditions on the components of vector fields $F$ can be given for constructing scalar functions $\alpha(x, y)$, which we call algebrizante factors, such that $\alpha F$ are algebrizable vector fields. Inverse integrating factors (see $[12,13]$ ) are constructed for these vector fields. 
2.3. Differentiability on Modules over Algebras. In this subsection we give the definition of $\mathbb{A}$-differentiability of functions with domain in $\mathbb{A} \times \mathbb{A}$ and image in $\mathbb{A}$.

The Cartesian product $\mathbb{A} \times \mathbb{A}$ defines a normed $\mathbb{A}$-module with respect to the norm $\|\cdot\|: \mathbb{A} \times \mathbb{A} \rightarrow \mathbb{R},\left\|\left(a_{1}, a_{2}\right)\right\|=$ $\sqrt{\left|a_{1}\right|^{2}+\left|a_{2}\right|^{2}}$. This norm satisfies

(i) $\|X\| \geq 0$ for all $X \in \mathbb{A} \times \mathbb{A}$ and $\|X\|=0$ if and only if $X=(0,0)$,

(ii) $\|a X\| \leq|a|\|X\|$ for all $a \in \mathbb{A}$ and $X \in \mathbb{A} \times \mathbb{A}$,

(iii) $\|X+Y\| \leq\|X\|+\|Y\|$ for all $X, Y \in \mathbb{A} \times \mathbb{A}$.

In the following definition $\|\cdot\|$ denotes the norm given above on the $\mathbb{A}$-module $\mathbb{A} \times \mathbb{A}$.

Definition 4. Let $\mathbb{A}$ be an algebra and $H: \Omega \subset \mathbb{A} \times \mathbb{A} \rightarrow$ A a function, where $\Omega$ is an open set. We say that $H$ is $\mathscr{H}(\mathbb{A})$-differentiable on $X_{0} \in \Omega$ if there exists a module homeomorphism $M: \mathbb{A} \times \mathbb{A} \rightarrow \mathbb{A}$, which we call the differential homomorphism of $H$ at $X_{0}$, which satisfies the condition

$$
\lim _{X \rightarrow X_{0}} \frac{\left\|H(X)-H\left(X_{0}\right)-M\left(X-X_{0}\right)\right\|}{\left\|X-X_{0}\right\|}=0 .
$$

We denote $M$ by $\mathscr{D} H\left(X_{0}\right)$. We say that $H$ is $\mathscr{H}(\mathbb{A})$ differentiable on $\Omega$ if $H$ is $\mathscr{H}(\mathbb{A})$-differentiable on all the points of $\Omega$.

A function $H: \Omega \subset \mathbb{A} \times \mathbb{A} \rightarrow \mathbb{A}$ is $\mathscr{H}(\mathbb{A})$-differentiable at $X_{0}$ if and only if the usual Jacobian matrix of $H: \Omega \subset$ $\mathbb{R}^{2} \times \mathbb{R}^{2} \rightarrow \mathbb{R}^{2}$ at $X_{0}$ satisfies $J H\left(X_{0}\right) \in R(\mathbb{A}) \times R(\mathbb{A})$. The differential homomorphism $\mathscr{D} H\left(X_{0}\right)$ is represented by a matrix in $M_{1,2}(\mathbb{A})$ with respect to the standard basis of $\mathbb{A} \times \mathbb{A}$, where $M_{1,2}(\mathbb{A})$ is the $\mathbb{A}$-module of all the matrices of one row and two columns with entries in $\mathbb{A}$; see [14].

\section{On Algebrizable Liftings of Functions $p: I \quad \subset$ $\mathbb{R} \rightarrow \mathbb{R}^{2}$}

In this section are considered functions $p: I \subset \mathbb{R} \rightarrow \mathbb{R}^{2}$ defined in open intervals $I$, and conditions for the existence of algebras $\mathbb{A}$ and $\mathbb{A}$-algebrizable functions $P$ such that $p(t)=$ $P(t e)$ will be determined, where $e$ is the unit of $\mathbb{A}$.

Definition 5. Let $p: I \subset \mathbb{R} \rightarrow \mathbb{R}^{2}$ be a function defined in an open interval $I$ and $\mathbb{A}$ an algebra with unit $e$. We will say that $P: V \subset \mathbb{R}^{2} \rightarrow \mathbb{R}^{2}$ is an $\mathbb{A}$-algebrizable lifting of $p$ if (a) $V$ is an open set on which $P$ is $\mathbb{A}$-algebrizable, (b) $\{$ te $: t \in I\} \subset V$, and (c) $p(t)=P(t e)$ for all $t \in I$.

As a consequence of the following proposition, the family of all the functions $p: I \subset \mathbb{R} \rightarrow \mathbb{R}^{2}$ having algebrizable liftings is an infinite dimensional algebra.

Proposition 6. Let $\mathbb{A}$ be an algebra and let $p, q: I \subset \mathbb{R} \rightarrow$ $\mathbb{R}^{2}$ be functions, where $I$ is an interval.

(a) Every constant function $p(t)=c$ admits the $\mathbb{A}$ algebrizable lifting $P(\tau)=c$. (b) $p(t)=$ te admits the $\mathbb{A}$-algebrizable lifting $P(\tau)=\tau$, where $\in \mathbb{A}$ is the unit of $\mathbb{A}$.

(c) If $p$ and $q$ admit $\mathbb{A}$-algebrizable liftings $P$ and $Q$ with respect to $\mathbb{A}$, respectively, and $a, b$ are constants in $\mathbb{A}$, then $a p+b q$ and $p q$ (all products with respect to $\mathbb{A}$ ) admit algebrizable liftings $a P+b Q$ and $P Q$, respectively.

(d) If $p$ has an $\mathbb{A}$-algebrizable lifting $P$ and $Q$ is an $\mathbb{A}$ algebrizable function with $\operatorname{Im}(P) \subset \operatorname{Dom}(Q)$, then $Q \circ P$ is an algebrizable lifting of $Q \circ p$.

Proof. Identity and constant functions are $\mathbb{A}$-differentiable for any algebra $\mathbb{A}$. Thus, (a) and (b) hold.

Let $p$ and $q$ be functions with $\mathbb{A}$-algebrizable liftings $P$ being $Q$ and $a, b \in \mathbb{A}$ constants. The functions $S=a P+b Q$ and $T=P Q$ are $\mathbb{A}$-algebrizable and satisfy

$$
\begin{aligned}
& S(t e)=a P(t e)+b Q(t e)=a p(t)+b q(t), \\
& T(t e)=P(t e) Q(t e)=p(t) q(t) .
\end{aligned}
$$

Therefore $S$ and $T$ are algebrizable liftings of $a p+b q$ and $p q$, respectively.

If $p$ has an $\mathbb{A}$-algebrizable lifting $P$ and $Q$ is an $\mathbb{A}$ differentiable function with $\operatorname{Im}(P) \subset \operatorname{Dom}(Q)$, then $Q$ 。 $P(t e)=Q \circ p(t)$. Therefore $Q \circ P$ is an $\mathbb{A}$-algebrizable lifting of $Q \circ p$.

Corollary 7. Let $\mathbb{A}$ be an algebra. Then the following functions admit $\mathbb{A}$-algebrizable liftings: polynomial functions, rational functions, trigonometric functions, exponential functions, and all of those functions which can be defined by linear combinations, products, quotients, and compositions of functions admitting algebrizable liftings.

Every function $t \mapsto(h(t), k(t))$ with polynomial components $h(t)$ and $k(t)$ has an $\mathbb{A}$-algebrizable lifting. The following proposition gives a wider class of these functions.

Proposition 8. Let $\mathbb{A}$ be an algebra. Any function $t \mapsto$ $(h(t), k(t))$ with components of the form

$$
\begin{aligned}
& h(t)=\frac{a_{m}}{t^{m}}+\frac{a_{m-1}}{t^{m-1}}+\cdots+\frac{a_{-1}}{t}+a_{0}+a_{1} t+\cdots a_{n} t^{n}, \\
& k(t)=\frac{b_{m}}{t^{m}}+\frac{b_{m-1}}{t^{m-1}}+\cdots+\frac{b_{-1}}{t}+b_{0}+b_{1} t+\cdots b_{n} t^{n},
\end{aligned}
$$

$m, n \in \mathbb{N}$, has an $\mathbb{A}$-algebrizable lifting. An $\mathbb{A}$-algebrizable lifting is given by

$$
\begin{aligned}
\tau \mapsto & \left(a_{m}, b_{m}\right) \frac{1}{\tau^{m}}+\cdots+\left(a_{-1}, b_{-1}\right) \frac{1}{\tau}+\left(a_{0}, b_{0}\right) \\
& +\left(a_{1}, b_{1}\right) \tau+\cdots+\left(a_{n}, b_{n}\right) \tau^{n} .
\end{aligned}
$$

Proof. Consider Q given by the above expression; then

$$
\left(a_{k}, b_{k}\right)(t e)^{k}=\left(a_{k}, b_{k}\right) t^{k} e=\left(a_{k} t^{k}, b_{k} t^{k}\right)
$$

holds for $k=-m, \ldots, n$, so $Q(t e)=(h(t), q(t))$, where $e$ is the unit of $\mathbb{A}$. Therefore, $Q$ is an $\mathbb{A}$-algebrizable lifting of $t \mapsto$ $(h(t), k(t))$. 
In particular, every function $p=\left(p_{1}, p_{2}\right): I \subset \mathbb{R} \rightarrow$ $\mathbb{R}^{2}$ with quadratic components $p_{1}(t)=a_{0}+a_{1} t+a_{2} t^{2}$ and $p_{2}(t)=b_{0}+b_{1} t+b_{2} t^{2}$ admits the $\mathbb{A}$-algebrizable lifting $P(\tau)=$ $\left(a_{0}, b_{0}\right)+\left(a_{1}, b_{1}\right) \tau+\left(a_{2}, b_{2}\right) \tau^{2}$.

\section{On Algebrizable Liftings of Planar Systems}

Solutions of every algebrizable planar system can be found by solving an algebrization of the system, as it is seen in the following proposition.

Proposition 9. If (2) is an algebrization of (1) and $\xi(\tau) a$ solution of (2), then $(x(t), y(t))=\xi(t e)$ is a solution of (1), where e denotes the unit of the corresponding algebra.

Proof. Let $\xi$ be a solution of (2). The derivative of $(x(t), y(t))=\xi(t e)$ with respect to $t$ is given by

$$
\begin{aligned}
\frac{d}{d t} & (x(t), y(t))=\frac{d}{d t} \xi(t e)=\left[\left.\frac{d \xi(\tau, \xi)}{d \tau}\right|_{\tau=(t e)} \frac{d \tau}{d t}\right] \\
& =\left.H(\tau, \xi(\tau))\right|_{\tau=(t e)} e=H(t e, \xi(t e)) \\
& =(f(t, x(t), y(t)), g(t, x(t), y(t))) .
\end{aligned}
$$

Thus, $(x(t), y(t))$ is a solution of system (1).

As a consequence of the following proposition, the family of all the functions $F: \Omega \subset \mathbb{R}^{3} \rightarrow \mathbb{R}^{2}$ having algebrizable liftings defines an infinite dimensional algebra.

Proposition 10. Let $\mathbb{A}$ be an algebra with unit e. In the following statements $F$ and $G$ denote functions defined on open sets $\Omega \subset \mathbb{R}^{3}$ and they have values in $\mathbb{R}^{2}$.

(a) $F(t, x, y)=c$ admits the $\mathscr{H}(\mathbb{A})$-differentiable lifting $H(\tau, \xi)=c$.

(b) $F(t, x, y)=$ te admits the $\mathscr{H}(\mathbb{A})$-differentiable lifting $H(\tau, \xi)=\tau$.

(c) $F(t, x, y)=(x, y)$ admits the $\mathscr{H}(\mathbb{A})$-differentiable lifting $H(\tau, \xi)=\xi$.

(d) If $F$ and $G$ admit $\mathscr{H}(\mathrm{A})$-differentiable liftings $H_{F}$ and $H_{G}$, respectively, and $a, b$ are constants in $\mathrm{A}$, then $\mathrm{H}_{F}+$ $b H_{G}$ and $F G$ (all products with respect to $\mathbb{A}$ ) have $\mathscr{H}(\mathrm{A})$-differentiable liftings $a H_{F}+b H_{G}$ and $H_{F} H_{G}$, respectively.

(e) Every function $E$ having the form $E(t, x, y)=F(t, G(t$, $x, y)$ ), where $F$ and $G$ admit $\mathscr{H}(\mathbb{A})$-differentiable liftings $H_{F}$ and $H_{G}$, admits an $\mathscr{H}(\mathrm{A})$-differentiable lifting $H_{E}$ given by $H_{E}(\tau, \xi)=H_{F}\left(\tau, H_{G}(\tau, \xi)\right)$.

(f) Every function $E$ having the form $E(t, x, y)=$ $F(t, P(x, y))$, where $F$ has an $\mathscr{H}(\mathbb{A})$-differentiable lifting $H_{F}$ and $P$ is an $\mathbb{A}$-differentiable function, admits an $\mathscr{H}(\mathbb{A})$-differentiable lifting $H_{E}(\tau, \xi)=H_{F}(\tau, P(\xi))$.
Proof. The proofs of (a), (b), and (c) are trivial. Let $H_{F}$ and $H_{G}$ be the algebrizable liftings of $F$ and $G$ and then $H_{F}+H_{G}$, $H_{F} H_{G}$, and $H_{F} / H_{G}$ are $\mathbb{A}$-algebrizable and

(i) $\left(a H_{F}+b H_{G}\right)(t e, x, y)=a H_{F}(t e, x, y)+b H_{G}(t e, x, y)=$ $a F(t, x, y)+a G(t, x, y)$,

(ii) $\left(H_{F} H_{G}\right)(t e, x, y)=H_{F}(t e, x, y) H_{G}(t e, x, y)=$ $F(t, x, y) G(t, x, y)$,

(iii) $H_{E}(t e, x, y)=H_{F}\left(t e, H_{G}(t e, x, y)\right)=F(t, G(t, x, y))$,

(iv) $H_{E}(t e, x, y)=H_{F}(t e, P(e, y))=F(t, P(x, y))$.

Thus, the proof is complete.

Corollary 11. Let $\mathbb{A}$ be an algebra. The following functions admit $\mathscr{H}(\mathbb{A})$-differentiable liftings: polynomial functions, rational functions, trigonometric functions, exponential functions, and all of those functions which can be defined by linear combinations, products, quotients, and compositions of functions admitting $\mathscr{H}(\mathbb{A})$-differentiable liftings.

Given a function $F(t, x, y)=(f(t, x, y), g(t, x, y))$, where $f, g$ are polynomial functions of the variables $x, y$, the goal of the paper is to determine if $F$ has an $\mathscr{H}(\mathbb{A})$ differentiable lifting. As a consequence of the following theorem, every function $F$ which is polynomial of the variables $t, x$, and $y$, has an $\mathscr{H}(\mathbb{A})$-differentiable lifting when $(x, y) \mapsto(f(t, x, y), g(t, x, y))$ is $\mathbb{A}$-differentiable for all $t$.

Theorem 12. Let $\mathbb{A}$ be an algebra with unit $e$ and $F$ : $\Omega \subset \mathbb{R}^{3} \rightarrow \mathbb{R}^{2}, F=(f, g)$, where $f=\sum_{k=0}^{m} f_{k}$, $g=\sum_{k=0}^{m} g_{k}$, and $f_{k}(t, x, y), g_{k}(t, x, y)$ are homogeneous polynomials of degree $k$ in the variables $x, y$, and $\Omega=I \times$ $\mathbb{R}^{2}$ for an open interval $I$. Then the following statements are equivalent.

(a) F has an $\mathscr{H}$ (A)-differentiable lifting $H$.

(b) The map $(x, y) \mapsto F(t, x, y)$ is $\mathbb{A}$-algebrizable for all $t \in I$ and the functions $h_{k}$ given by $h_{k}(t)=$ $\left(f_{k}(t, e), g_{k}(t, e)\right)$ have A-algebrizable liftings, for $k=$ $0,1, \ldots, m$.

(c) $H(\tau, \xi)=\sum_{k=0}^{m} H_{k}(\tau) \xi^{k}$ is an $\mathscr{H}(\mathbb{A})$-differentiable lifting of $F$, where $H_{k}(\tau)$ are $\mathbb{A}$-algebrizable liftings of $h_{k}(t)=\left(f_{k}(t, e), g_{k}(t, e)\right)$.

Proof. Obviously (a) implies (b) and (c) implies (a). We now show that (b) implies (c). Suppose that $f(t, x, y)$ and $g(t, x, y)$ are homogenous polynomials $f(t, x, y)=\sum_{k=0}^{n} p_{k}(t) x^{k} y^{n-k}$ and $g(t, x, y)=\sum_{k=0}^{n} q_{k}(t) x^{k} y^{n-k}$ of the variables $x, y$ defined 
on the set $\Omega=I \times \mathbb{R}^{2}$ as above. Taking the partial derivatives of $F$ with respect to $x$ and $y$ we obtain

$$
\begin{aligned}
& \frac{\partial(f, g)}{\partial(x, y)}(t, x, y) \\
& =\left(\begin{array}{ll}
\sum_{k=1}^{n} k p_{k}(t) x^{k-1} y^{n-k} & \sum_{k=1}^{n}(n-k+1) p_{k-1}(t) x^{k-1} y^{n-k} \\
\sum_{k=1}^{n} k q_{k}(t) x^{k-1} y^{n-k} & \sum_{k=1}^{n}(n-k+1) q_{k-1}(t) x^{k-1} y^{n-k}
\end{array}\right) \\
& =\left(\begin{array}{ll}
\sum_{k=1}^{n} k p_{k}(t) x^{k-1} y^{n-k} & \sum_{k=1}^{n}(n-k+1) p_{k-1}(t) x^{k-1} y^{n-k} \\
\sum_{k=1}^{n} k q_{k}(t) x^{k-1} y^{n-k} & \sum_{k=1}^{n}(n-k+1) q_{k-1}(t) x^{k-1} y^{n-k}
\end{array}\right) \\
& =\sum_{k=1}^{n} x^{k-1} y^{n-k}\left(\begin{array}{ll}
k p_{k}(t) & (n-k+1) p_{k-1}(t) \\
k q_{k}(t) & (n-k+1) q_{k-1}(t)
\end{array}\right) .
\end{aligned}
$$

Let $R$ be the first fundamental representation of $\mathbb{A}$. Since $(x, y) \mapsto(f(t, x, y), g(t, x, y))$ is $\mathbb{A}$-algebrizable for all $t \in I$, then $(\partial(f, g) / \partial(x, y))(t, x, y) \in R(\mathbb{A})$ for all $(t, x, y) \in \Omega$. Thus

$$
\frac{\partial(f, g)}{\partial(x, y)}(t, 0,1)=\left(\begin{array}{ll}
p_{1}(t) & n p_{0}(t) \\
q_{1}(t) & n q_{0}(t)
\end{array}\right) \in R(\mathbb{A})
$$

for all $t \in I$ and then

$$
\begin{aligned}
& \frac{\partial(f, g)}{\partial(x, y)}(t, x, y)-y^{n-1}\left(\begin{array}{ll}
p_{1}(t) & n p_{0}(t) \\
q_{1}(t) & n q_{0}(t)
\end{array}\right) \\
& \quad=\sum_{k=2}^{n} x^{k-1} y^{n-k}\left(\begin{array}{ll}
k p_{k}(t) & (n-k+1) p_{k-1}(t) \\
k q_{k}(t) & (n-k+1) q_{k-1}(t)
\end{array}\right)
\end{aligned}
$$

is in $R(\mathbb{A})$ for all $(t, x, y) \in \Omega$. If $x(s)=1 / s$ and $y(s)=\sqrt[(n-2)]{s}$, then

$$
\begin{aligned}
\lim _{s \rightarrow \infty} & {\left[\frac{\partial(f, g)}{\partial(x, y)}(t, x(s), y(s))\right.} \\
- & {\left.[y(s)]^{n-1}\left(\begin{array}{cc}
p_{1}(t) & n p_{0}(t) \\
q_{1}(t) & n q_{0}(t)
\end{array}\right)\right] } \\
= & \left(\begin{array}{ll}
p_{2}(t) & (n-1) p_{1}(t) \\
q_{2}(t) & (n-1) q_{1}(t)
\end{array}\right) \in R(\mathbb{A}) .
\end{aligned}
$$

Following the same idea, for $k=1,2, \ldots, n$

$$
\left(\begin{array}{ll}
k p_{k}(t) & (n-k+1) p_{k-1}(t) \\
k q_{k}(t) & (n-k+1) q_{k-1}(t)
\end{array}\right) \in R(\mathbb{A}) .
$$

If $(x, y) \mapsto(f(t, x, y), g(t, x, y))$ is $\mathbb{A}$-algebrizable for an algebra $\mathbb{A}$ with Type (I) product (given in Section 2), then

$$
\begin{array}{r}
k p_{k}+a k q_{k}-(n-k+1) q_{k-1}=0 \\
b k q_{k}+(n-k+1) p_{k-1}=0
\end{array}
$$

or equivalently

$$
\left(q_{k-1}, p_{k-1}\right)=\left(\frac{k p_{k}+a k q_{k}}{n-k+1},-\frac{b k q_{k}}{n-k+1}\right)
$$

for $k=1, \ldots, n$. Thus, the functions $p_{k}, q_{k}$ are determined by $p_{n}, q_{n}$ for $k=0,1, \ldots, n-1$. Since $(f(t, 1,0), g(t, 1,0))=\left(p_{n}(t), q_{n}(t)\right)$, then $(f(t, x, y), g(t, x, y))=\left(p_{n}(t), q_{n}(t)\right)(x, y)^{n}$.

Therefore, $F$ has an $\mathscr{H}(\mathbb{A})$-differentiable lifting $H(\tau, \xi)=$ $H_{n}(\tau) \xi^{n}$, where $H_{n}(\tau)$ is an $\mathbb{A}$-algebrizable lifting of $t \mapsto$ $\left(p_{n}(t), q_{n}(t)\right)$.

If $(x, y) \mapsto(f(t, x, y), g(t, x, y))$ is A-algebrizable for an algebra $\mathbb{A}$ with Type (II) product (given in Section 2 ), then

$$
\begin{aligned}
k p_{k}+a(n-k+1) p_{k-1}-(n-k+1) q_{k-1} & =0 \\
k q_{k} & =0
\end{aligned}
$$

or equivalently

$$
\left(p_{k}, q_{k}\right)=\left(-\frac{a(n-k+1) p_{k-1}}{k}, 0\right)
$$

for $k=1, \ldots, n$. Thus, $q_{k}=0$ and $p_{k}$ is determined by $p_{0}$, for $k=1,2, \ldots, n$. Since $(f(t, 0,1), g(t, 0,1))=\left(p_{0}(t), q_{0}(t)\right)$, then $(f(t, x, y), g(t, x, y))=\left(p_{0}(t), q_{0}(t)\right)(x, y)^{n}$.

Therefore, $F$ has an $\mathscr{H}(\mathbb{A})$-differentiable lifting $H(\tau, \xi)=$ $H_{n}(\tau) \xi^{n}$, where $H_{n}(\tau)$ is an $\mathbb{A}$-algebrizable lifting of $t \mapsto$ $\left(p_{0}(t), q_{0}(t)\right)$.

If $(x, y) \mapsto(f(t, x, y), g(t, x, y))$ is $\mathbb{A}$-algebrizable for an algebra $\mathbb{A}$ with Type (III) product (given in Section 2), then $(n-k+1) p_{k-1}=0$ and $k q_{k}=0$; that is, $p_{k-1}=0, q_{k}=0$, for $k=1, \ldots, n$. Thus, $q_{k}=0$ and $p_{k}$ is determined by $p_{0}$, for $k=1,2, \ldots, n$. Since $(f(t, 0,1), g(t, 0,1))=\left(p_{n}(t), q_{0}(t)\right)$, then $(f(t, x, y), g(t, x, y))=\left(p_{n}(t), q_{0}(t)\right)(x, y)^{n}$.

Therefore, $F$ has an $\mathscr{H}(\mathbb{A})$-differentiable lifting $H(\tau, \xi)=$ $H_{n}(\tau) \xi^{n}$, where $H_{n}(\tau)$ is an $\mathbb{A}$-algebrizable lifting of $t \mapsto$ $\left(p_{n}(t), q_{0}(t)\right)$.

Thus, if $f$ and $g$ are homogenous polynomial of degree $n$ in the variables $x$ and $y$, then $H(\tau, \xi)=H_{n}(\tau) \xi^{n}$ in each of the cases of algebras defined by products of Types (I), (II), and (III) given in Section 2. Since a polynomial function $(t, x, y) \mapsto(f(t, x, y), g(t, x, y))$ in the variables $x$ and $y$ can be seen as the finite addition of homogenous polynomial in the variables $x$ and $y$, by (d) of Proposition 10, (b) implies (c).

Example 13. Consider the planar system (6); then

$$
\begin{aligned}
& F(t, x, y) \\
& \quad=\left(-\frac{1}{t^{3}}-\frac{2 x}{t}+t x^{2}-t y^{2},-\frac{2 y}{t}+2 t x y+t y^{2}\right) .
\end{aligned}
$$

The Jacobian $\partial F / \partial(x, y)$ is given by

$$
\frac{\partial F(t, x, y)}{\partial(x, y)}=\left(\begin{array}{cc}
-\frac{2}{t}+2 t x & -2 t y \\
2 t y & -\frac{2}{t}+2 t x+2 t y
\end{array}\right) .
$$


Thus, $\partial F / \partial(x, y)$ is orthogonal to the matrices

$$
\begin{aligned}
& B_{1}=\left(\begin{array}{cc}
1 & 0 \\
1 & -1
\end{array}\right), \\
& B_{2}=\left(\begin{array}{ll}
0 & 1 \\
1 & 0
\end{array}\right),
\end{aligned}
$$

for all $(x, y)$; that is, $\left\langle\partial F / \partial(x, y), B_{i}\right\rangle=0$ for $i=1,2$. The map $(x, y) \mapsto F(t, x, y)$ is $\mathbb{A}$-algebrizable for an algebra of Type (I) with constants $a=1$ and $b=1$; see Section 2 . The function $F$ can be written as

$$
\begin{aligned}
F(t, x, y)= & \left(-\frac{1}{t^{3}}, 0\right)+\left(-\frac{2}{t} x,-\frac{2}{t} y\right) \\
& +\left(t x^{2}-t y^{2}, t y^{2}\right) .
\end{aligned}
$$

Thus, the functions $p_{i}$ and $q_{i}$ of Theorem 12 are given by $p_{0}(t, x, y)=-1 / t^{3}, q_{0}(t, x, y)=0, p_{1}(t, x, y)=-(2 / t) x$, $q_{1}(t, x, y)=-(2 / t) y, p_{2}(t, x, y)=t x^{2}-t y^{2}$, and $q_{2}(t, x, y)=$ $t y^{2}$.

The unit $e$ of $\mathbb{A}$ is $e=(1,0)$ and then $h_{0}(t)=\left(-1 / t^{3}, 0\right)$, $h_{1}(t)=(-2 / t, 0)$, and $h_{2}(t)=(t, 0)$ have $\mathbb{A}$-algebrizable liftings $H_{0}(\tau)=-1 / \tau^{3}, H_{1}(\tau)=-2 / \tau$, and $H_{2}(\tau)=\tau$, respectively.

From the form of $F(t, x e)=\left(-1 / t^{3}-(2 / t) x-t x^{2}\right) e, H$ must be

$$
H(\tau, \xi)=-\frac{1}{\tau^{3}}-\frac{2}{\tau} \xi+\tau \xi^{2}
$$

\section{The Case of Second-Degree Polynomials in the Variables $t, x$, and $y$}

If $f$ and $g$ in (1) are quadratic polynomials in three variables $t, x$, and $y$ and $\mathbb{A}$ is an algebra with respect to which the map $(x, y) \mapsto(f(t, x, y), g(t, x, y))$ is $\mathbb{A}$-algebrizable, it will be showed that the $\mathscr{H}(\mathbb{A})$-differentiable lifting $H$ of $F=(f, g)$ is a polynomial in two variables of $\mathbb{A}$. Under these conditions (1) has an algebrization which is a Riccati equation over $\mathbb{A}$ having the form

$$
\frac{d \xi}{d \tau}=P(\tau)+Q(\tau) \xi+R(\tau) \xi^{2}
$$

where $P, Q$, and $R$ are polynomials over $\mathbb{A}$ of degrees two, one, and zero, respectively.

Consider system (1) where $f, g: \Omega \subset \mathbb{R}^{3} \rightarrow \mathbb{R}^{2}$ are second-degree polynomials of three variables $t, x$, and $y$; that is,

$$
\begin{aligned}
f(t, x, y)= & a_{0}+a_{1} t+a_{2} x+a_{3} y+a_{4} t^{2}+a_{5} t x \\
& +a_{6} t y+a_{7} x^{2}+a_{8} x y+a_{9} y^{2} \\
g(t, x, y)= & b_{0}+b_{1} t+b_{2} x+b_{3} y+b_{4} t^{2}+b_{5} t x+b_{6} t y \\
& +b_{7} x^{2}+b_{8} x y+b_{9} y^{2} .
\end{aligned}
$$

All the quadratic vector fields which are algebrizable with respect to algebras with Type (I) products have the form

$$
\begin{aligned}
\frac{d x}{d t}= & A_{0}+\left(B_{2}-a B_{1}\right) x-b B_{1} y+\left(\frac{1}{2} B_{4}-a B_{3}\right) x^{2} \\
& -2 b B_{3} x y-\frac{1}{2} b B_{4} y^{2}, \\
\frac{d y}{d t}= & B_{0}+B_{1} x+B_{2} y+B_{3} x^{2}+B_{4} x y \\
& +\left(\frac{1}{2} a B_{4}-b B_{3}\right) y^{2},
\end{aligned}
$$

where $a, b, A_{0}, B_{0}, B_{1}, \ldots, B_{5}$ are real constants; see Section 2.2. The algebrizability of $(x, y) \mapsto$ $(f(t, x, y), g(t, x, y))$ with respect to algebras with Type (I) products can be verified, by considering $t$ as a constant.

The following theorems give conditions that characterize the algebrizability of planar systems like (1) when $f$ and $g$ are quadratic polynomials. The algebrizability of nonautonomous quadratic systems with respect to algebras with Type (I) products is given in the following theorem.

Theorem 14. Let $\mathbb{A}$ be an algebra with Type (I) product defined by constants $a$ and $b$ and $f, g$ the polynomials (35). The following statements are equivalent.

(1) The map $F:(x, y) \mapsto(f(t, x, y), g(t, x, y))$ is $\mathbb{A}$ algebrizable.

(2) The functions $f$ and $g$ have the form

$$
\begin{aligned}
f(t, x, y)= & a_{0}+a_{1} t+\left(b_{3}-a b_{2}\right) x-b b_{2} y+a_{4} t^{2} \\
& +\left(b_{6}-a b_{5}\right) t x-b b_{5} t y \\
& +\left(\frac{b_{8}}{2}-a b_{7}\right) x^{2}-2 b b_{7} x y-\frac{b b_{8}}{2} y^{2}, \\
g(t, x, y)= & b_{0}+b_{1} t+b_{2} x+b_{3} y+b_{4} t^{2}+b_{5} t x+b_{6} t y \\
& +b_{7} x^{2}+b_{8} x y+\left(\frac{a b_{8}}{2}-b b_{7}\right) y^{2} .
\end{aligned}
$$

(3) The function $H: \mathbb{A} \times \mathbb{A} \rightarrow \mathbb{A}$ of the variables $\tau=(t, s)$ and $\xi=(x, y)$, defined by

$$
H(\tau, \xi)=A_{0}+A_{1} \tau+A_{2} \xi+A_{3} \tau^{2}+A_{4} \tau \xi+A_{5} \xi^{2},
$$

is an $\mathscr{H}(\mathbb{A})$-differentiable lifting of $F$, where

$$
\begin{aligned}
& A_{0}=\left(a_{0}, b_{0}\right), \\
& A_{1}=\left(a_{1}, b_{1}\right), \\
& A_{2}=\left(b_{3}-a b_{2}, b_{2}\right), \\
& A_{3}=\left(a_{4}, b_{4}\right), \\
& A_{4}=\left(b_{6}-a b_{5}, b_{5}\right), \\
& A_{5}=\left(\frac{b_{8}}{2}-a b_{7}, b_{7}\right) .
\end{aligned}
$$


Proof. Writing $g(t, x, y)=B_{0}+B_{1} x+B_{2} y+B_{3} x^{2}+B_{4} x y+B_{5} y^{2}$ yields

$$
\begin{aligned}
& B_{0}=b_{0}+b_{1} t+b_{4} t^{2}, \\
& B_{1}=b_{2}+b_{5} t, \\
& B_{2}=b_{3}+b_{6} t, \\
& B_{3}=b_{7}, \\
& B_{4}=b_{8}, \\
& B_{5}=b_{9} .
\end{aligned}
$$

The function $(x, y) \mapsto(f(t, x, y), g(t, x, y))$ is $\mathbb{A}$-algebrizable if and only if

$$
\begin{aligned}
f(t, x, y)= & A_{0}+\left(B_{2}-a B_{1}\right) x-b B_{1} y \\
& +\left(\frac{1}{2} B_{4}-a B_{3}\right) x^{2}-2 b B_{3} x y-\frac{1}{2} b B_{4} y^{2},
\end{aligned}
$$

where $A_{0}=a_{0}+a_{1} t+a_{4} t^{2}$ and $b_{9}=a b_{8} / 2-b b_{7}$. Thus, statements (1) and (2) are equivalent.

The function $H$ is polynomial in the variables $\tau$ and $\xi$ of $\mathbb{A}$; hence $H$ is $\mathscr{H}(\mathbb{A})$-differentiable. $H$ satisfies $H(t e, x, y)=$ $(f(t, x, y), g(t, x, y))$, where $e$ is the unit of $\mathbb{A}$. So, $H$ is an $\mathscr{H}(\mathbb{A})$-differentiable lifting of $F$. Thus, statement (2) implies statement (3).

Since $H$ is $\mathscr{H}(\mathbb{A})$-differentiable and $F(t, x, y)=$ $H(t e, x, y)$, then $(x, y) \mapsto F(t, x, y)$ is $\mathbb{A}$-algebrizable for all $t$. Thus, statement (3) implies statement (1).

All the quadratic vector fields which are algebrizable with respect to algebras with Type (II) products have the form

$$
\begin{aligned}
& \dot{x}=A_{0}+A_{1} x+A_{2} y-\frac{1}{2} a A_{3} x^{2}+A_{3} x y+A_{4} y^{2}, \\
& \dot{y}=B_{0}+\left(A_{1}+a A_{2}\right) y+\left(\frac{1}{2} A_{3}+a A_{4}\right) y^{2},
\end{aligned}
$$

where $a, A_{0}, \ldots, A_{4}, B_{0}$ are real constants; see Section 2.2. The algebrizability of $(x, y) \mapsto(f(t, x, y), g(t, x, y))$ with respect to algebras with Type (II) products can be verified, by considering $t$ as a constant.

The algebrizability of nonautonomous quadratic systems with respect to algebras with Type (II) products is given in the following theorem.

Theorem 15. Let $\mathbb{A}$ be an algebra with Type (II) product defined by the constant $a$ and let $f, g$ be the polynomials (35). The following statements are equivalent.

(1) The map $(x, y) \mapsto(f(t, x, y), g(t, x, y))$ is $\mathbb{A}$ algebrizable.
(2) The functions $f$ and $g$ are given by

$$
\begin{aligned}
f(t, x, y)= & a_{0}+a_{1} t+a_{2} x+a_{3} y+a_{4} t^{2}+a_{5} t x \\
& +a_{6} t y-\frac{1}{2} a a_{8} x^{2}+a_{8} x y+a_{9} y^{2}, \\
g(t, x, y)= & b_{0}+b_{1} t+\left(a_{2}+a a_{3}\right) y+b_{4} t^{2} \\
& +\left(a_{5}+a a_{6}\right) t y+\left(\frac{a_{8}}{2}+a a_{9}\right) y^{2} .
\end{aligned}
$$

(3) The function $H: \mathbb{A} \times \mathbb{A} \rightarrow \mathbb{A}$ of the variables $\tau=(t, s)$ and $\xi=(x, y)$, defined by

$$
H(\tau, \xi)=A_{0}+A_{1} \tau+A_{2} \xi+A_{3} \tau^{2}+A_{4} \tau \xi+A_{5} \xi^{2}
$$

is an $\mathscr{H}(\mathbb{A})$-differentiable lifting of $F$, where

$$
\begin{aligned}
& A_{0}=\left(a_{0}, b_{0}\right), \\
& A_{1}=\left(a_{1}, b_{1}\right), \\
& A_{2}=\left(a_{3}, a_{2}+a a_{3}\right), \\
& A_{3}=\left(a_{4}, b_{4}\right), \\
& A_{4}=\left(a_{6}, a_{5}+a a_{6}\right), \\
& A_{5}=\left(a_{9}, \frac{a_{8}}{2}+a a_{9}\right) .
\end{aligned}
$$

Proof. Writing $f(t, x, y)=A_{0}+A_{1} x+A_{2} y-(1 / 2) a A_{3} x^{2}+$ $A_{3} x y+A_{4} y^{2}$ yields

$$
\begin{aligned}
& A_{0}=a_{0}+a_{1} t+a_{4} t^{2}, \\
& A_{1}=a_{2}+a_{5} t, \\
& A_{2}=a_{3}+a_{6} t, \\
& A_{3}=a_{8}, \\
& A_{4}=a_{9},
\end{aligned}
$$

and the map $(x, y) \mapsto(f(t, x, y), g(t, x, y))$ is $\mathbb{A}$-algebrizable if and only if

$$
g(t, x, y)=B_{0}+\left(A_{1}+a A_{2}\right) y+\left(\frac{1}{2} A_{3}+a A_{4}\right) y^{2},
$$

where $B_{0}=b_{0}+b_{1} t+b_{4} t^{2}$. Thus, statements (1) and (2) are equivalent.

The rest of the proof is similar to that of Theorem 14.

All the quadratic vector fields which are differentiable with respect to algebras with Type (III) products have the form

$$
\begin{aligned}
& \dot{x}=A_{0}+A_{1} x+A_{2} x^{2}, \\
& \dot{y}=B_{0}+B_{1} y+B_{2} y^{2},
\end{aligned}
$$


where $A_{0}, B_{0}, A_{1}, B_{1}, A_{2}, B_{2}$ are real constants; see Section 2.2. The algebrizability of $(x, y) \mapsto$ $(f(t, x, y), g(t, x, y))$ with respect to algebras with Type (III) products can be verified, by considering $t$ as a constant.

The algebrizability of nonautonomous quadratic systems with respect to algebras with Type (III) products is given in the following theorem.

Theorem 16. Let $\mathbb{A}$ be an algebra with Type (III) product defined by the constant $a$ and let $f, g$ be the polynomials (35). The following statements are equivalent.

(1) The map $(x, y) \mapsto(f(t, x, y), g(t, x, y))$ is $\mathbb{A}$ algebrizable.

(2) The functions $f$ and $g$ are given by

$$
\begin{aligned}
& f(t, x, y)=a_{0}+a_{1} t+a_{2} x+a_{4} t^{2}+a_{5} t x+a_{7} x^{2}, \\
& g(t, x, y)=b_{0}+b_{1} t+b_{3} y+b_{4} t^{2}+b_{6} t y+b_{9} y^{2} .
\end{aligned}
$$

(3) The function $H: \mathbb{A} \times \mathbb{A} \rightarrow \mathbb{A}$ of the variables $\tau=(t, s)$ and $\xi=(x, y)$, defined by

$$
H(\tau, \xi)=A_{0}+A_{1} \tau+A_{2} \xi+A_{3} \tau^{2}+A_{4} \tau \xi+A_{5} \xi^{2},
$$

is an $\mathscr{H}(\mathbb{A})$-differentiable lifting of $F$, where $A_{0}=$ $\left(a_{0}, b_{0}\right), A_{1}=\left(a_{1}, b_{1}\right), A_{2}=\left(a_{2}, b_{3}\right), A_{3}=\left(a_{4}, b_{4}\right)$, $A_{4}=\left(a_{5}, b_{6}\right)$, and $A_{5}=\left(a_{7}, b_{9}\right)$.

Proof. Function $(x, y) \mapsto(f(t, x, y), g(t, x, y))$ is $\mathbb{A}$ algebrizable if and only if

$$
\begin{aligned}
& f(t, x, y)=a_{0}+a_{1} t+a_{2} x+a_{4} t^{2}+a_{5} t x+a_{7} x^{2}, \\
& g(t, x, y)=b_{0}+b_{1} t+b_{3} y+b_{4} t^{2}+b_{6} t y+b_{9} y^{2} .
\end{aligned}
$$

Thus, first and second statements are equivalents. The function $H$ given by $H(\tau, \xi)=A_{0}+A_{1} \tau+A_{2} \xi+A_{3} \tau^{2}+A_{4} \tau \xi+A_{5} \xi^{2}$, where $A_{0}=\left(a_{0}, b_{0}\right), A_{1}=\left(a_{1}, b_{1}\right), A_{2}=\left(a_{2}, b_{3}\right), A_{3}=\left(a_{4}, b_{4}\right)$, $A_{4}=\left(a_{5}, b_{6}\right), A_{5}=\left(a_{7}, b_{9}\right), \tau=(r, s)$, and $\xi=(x, y)$, satisfies $H(t e, x, y)=(f(t, x, y), g(t, x, y))$. Thus, second and third statements are equivalents.

By Theorems 14, 15, and 16, an algebrization of quadratic systems is a Riccati equation over an algebra $d \xi / d \tau=P(\tau)+$ $Q(\tau) \xi+R(\tau) \xi^{2}$, where $P(\tau)=A_{0}+A_{1} \tau+A_{3} \tau^{2}, Q(\tau)=$ $A_{2}+A_{4} \tau$, and $R(\tau)=A_{5}$. In the following example is given a nonautonomous quadratic system for the which an algebrization is found by using Theorem 14 .

Example 17. Consider system (1) given by

$$
\begin{aligned}
f(t, x, y)= & 1+7 t-x-3 y+5 t^{2}-t x-3 t y-6 x y \\
& -6 y^{2} \\
g(t, x, y)= & 1+t+x+y+t^{2}+t x+t y+x^{2}+4 x y \\
& +y^{2} .
\end{aligned}
$$

The matrices

$$
\begin{aligned}
& B_{1}=\left(\begin{array}{cc}
1 & 0 \\
2 & -1
\end{array}\right), \\
& B_{2}=\left(\begin{array}{ll}
0 & 1 \\
3 & 0
\end{array}\right)
\end{aligned}
$$

satisfy $\left\langle B_{i}, \partial(f, g) / \partial(x, y)\right\rangle=0$ for $i=1,2$. Thus, $F:(x, y) \mapsto$ $(f(t, x, y), g(t, x, y))$ is $\mathbb{A}$-algebrizable for an algebra $\mathbb{A}$ with Type (I) product defined by the constants $a=2$ and $b=3$.

The conditions of Theorem 14 are satisfied; then the $\mathscr{H}(\mathbb{A})$-differentiable lifting $H$ of $F$ can be written as

$$
H(\tau, \xi)=A_{0}+A_{1} \tau+A_{2} \xi+A_{3} \tau^{2}+A_{4} \tau \xi+A_{5} \xi^{2},
$$

where $A_{0}=(1,1), A_{1}=(7,1), A_{2}=(-1,1), A_{3}=(5,1)$, $A_{4}=(-1,1)$, and $A_{5}=(0,1)$.

\section{Disclosure}

The authors declare having no financial affiliation with any organization regarding the material discussed here.

\section{Conflict of Interests}

The authors declare that there is no conflict of interests concerning this text. The issues discussed in this paper do not have any secondary interest for any of the authors.

\section{Acknowledgments}

The authors wish to acknowledge the support of Grants Promep/103.5/13/ and CB-2010/150532 Conacyt.

\section{References}

[1] E. K. Blum, "A theory of analytic functions in Banach algebras," Transactions of the American Mathematical Society, vol. 78, no. 2, pp. 343-370, 1955.

[2] P. W. Ketchum, "Analytic functions of hypercomplex variables," Transactions of the American Mathematical Society, vol. 30, no. 4, pp. 641-641, 1928.

[3] E. R. Lorch, "The theory of analytic functions in normed abelian vector rings," Transactions of the American Mathematical Society, vol. 54, no. 3, pp. 414-425, 1943.

[4] J. A. Ward, "A theory of analytic functions in linear associative algebras," Duke Mathematical Journal, vol. 7, no. 1, pp. 233-248, 1940.

[5] J. A. Ward, "From generalized Cauchy-Riemann equations to linear algebra," Proceedings of the American Mathematical Society, vol. 4, no. 3, pp. 456-461, 1953.

[6] E. López-González, "Differential equations over algebras," Advances and Applications in Mathematical Sciences, vol. 8, no. 2, pp. 189-214, 2011.

[7] A. Alvarez-Parrilla, M. E. Frías-Armenta, E. López-González, and C. Yee-Romero, "On solving systems of autonomous ordinary differential equations by reduction to a variable of an algebra," International Journal of Mathematics and Mathematical Sciences, vol. 2012, Article ID 753916, 21 pages, 2012. 
[8] E. J. Wilczynski, "Review: Abraham Cohen, an introduction to the lie theory of one-parameter groups with applications to the solution of differential equations," Bulletin of the American Mathematical Society, vol. 18, no. 10, pp. 514-515, 1912, http://projecteuclid.org/euclid.bams/1183421829.

[9] J. M. Page, Ordinary Differential Equations with an Introduction to Lie's Theory or the Group of One Parameter, Macmillan Publishers, London, UK, 1897.

[10] R. A. Steinhour, The truth about lie symmetries: solving differential equations with symmetry methods [Senior Independent Study Theses], 2013, http://openworks.wooster.edu/ independentstudy/949.

[11] R. Pierce, Associative Algebras, Springer, New York, NY, USA, 1982.

[12] K. I. T. Al-Dosary, "Inverse integrating factor for classes of planar differential systems," International Journal of Mathematical Analysis, vol. 4, no. 29-32, pp. 1433-1446, 2010.

[13] I. A. García and M. Grau, "A Survey on the inverse integrating factor," Qualitative Theory of Dynamical Systems, vol. 9, no. 1-2, pp. 115-166, 2010.

[14] W. Brown, Matrices over Commutative Rings, Monographs and Textbooks in Pure and Applied Mathematics, Marcel Dekker, 1992. 


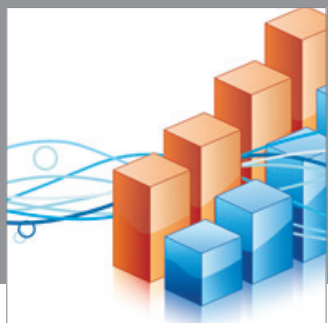

Advances in

Operations Research

mansans

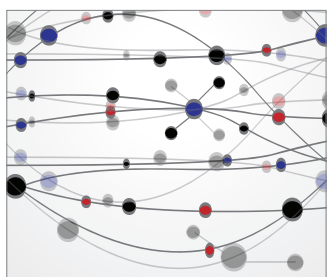

The Scientific World Journal
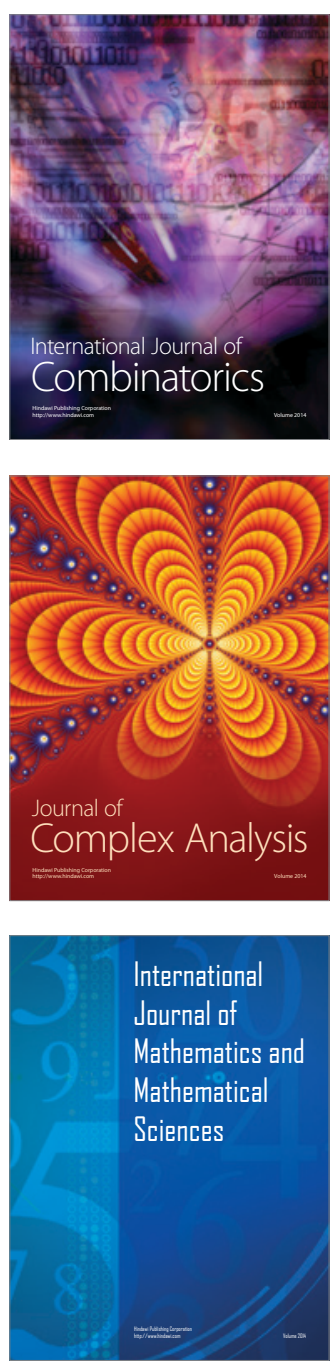
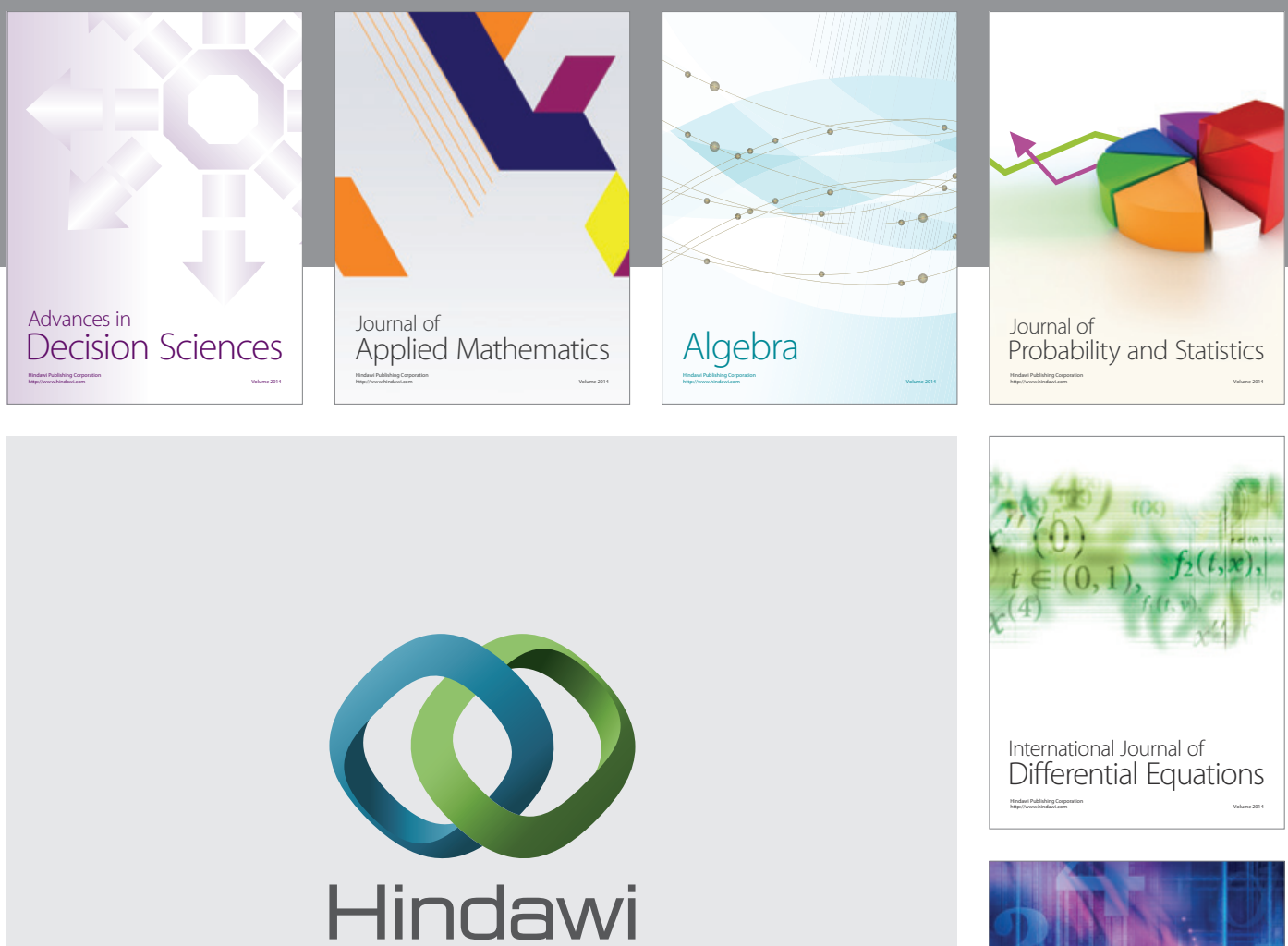

Submit your manuscripts at http://www.hindawi.com
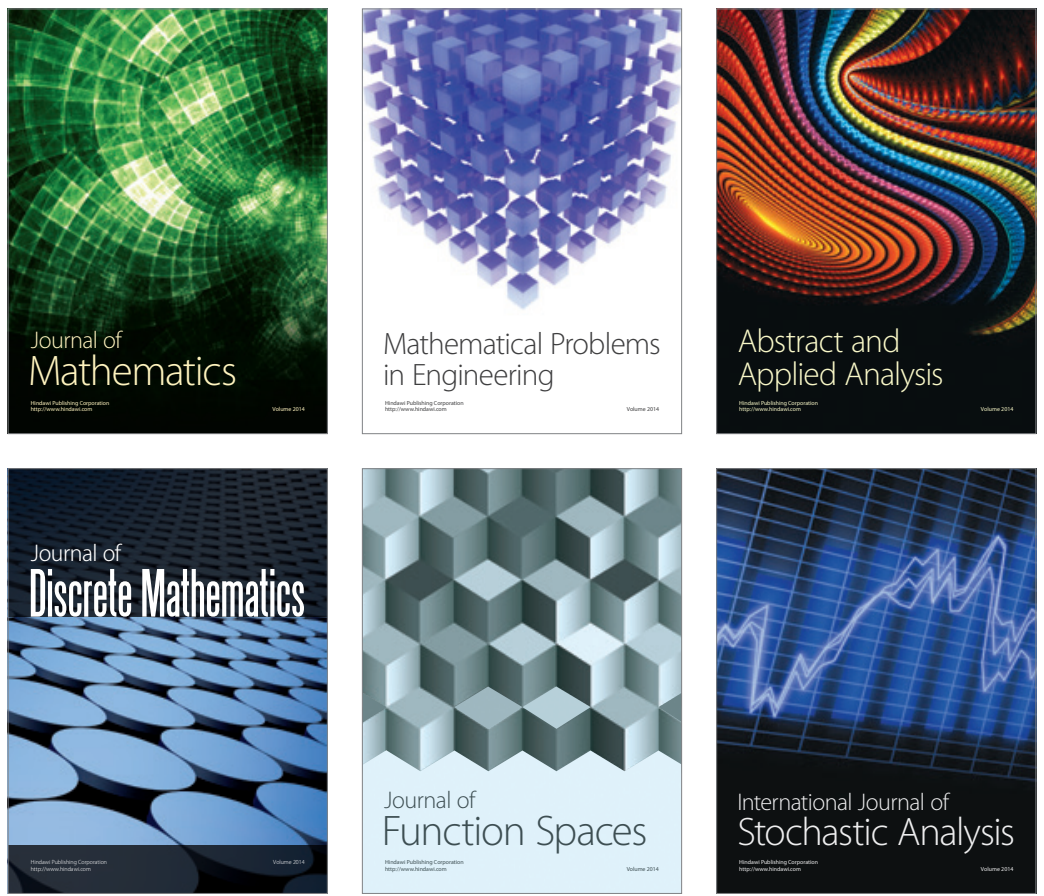

Journal of

Function Spaces

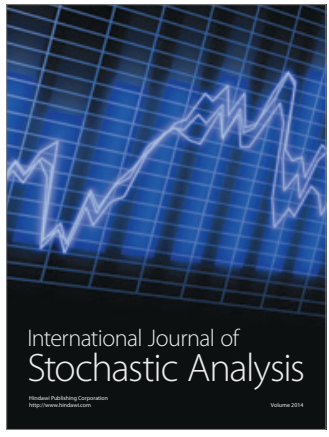

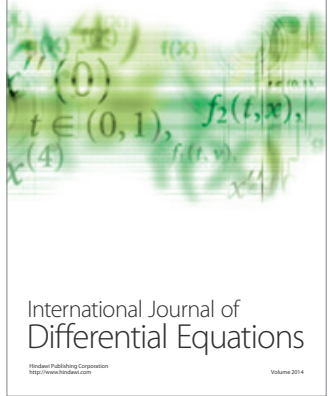
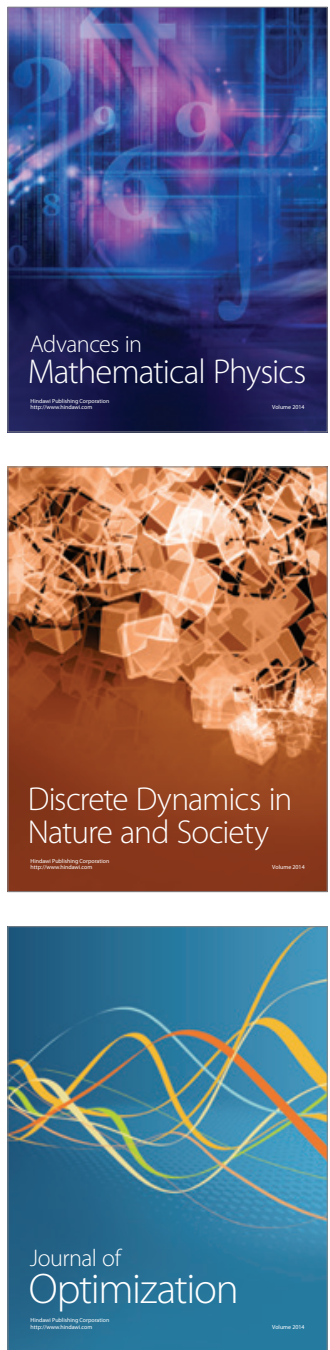